This article is licensed under the Creative Commons Attribution-NonCommercial 4.0 International License (CC BY-NC) (http://www.karger.com/Services/OpenAccessLicense). Usage and distribution for commercial purposes requires written permission.

\title{
Prognostic Discrepancy of the 6th and 7th UICC N Classification for Lymph Node Staging in Gastric Cancer Patients after Curative Resection
}

\author{
Sung Jin Oh ${ }^{\mathrm{a}, \mathrm{b}}$ Byoung Jo Suh ${ }^{\mathrm{a}, \mathrm{b}}$ Jong Kwon Park ${ }^{\mathrm{a}}$ Sung Don Oh \\ Hang Jong $\mathrm{Yu}^{\mathrm{c}}$ \\ ${ }^{a}$ Department of Surgery, Haeundae Paik Hospital, Busan, Republic of Korea; \\ ${ }^{b}$ Memorial Jin-Pok Kim Korea Gastric Cancer Center, Inje University College of Medicine, \\ Busan, Republic of Korea; 'Department of Surgery, Korea Cancer Center Hospital, \\ Seoul, Republic of Korea
}

\section{Keywords}

Gastric cancer · TNM system · Lymph node status

\begin{abstract}
Background: The validity of $\mathrm{N}$ classification of the 7th edition of the American Joint Committee on Cancer/Union Internationale contre le Cancer (AJCC/UICC) tumor-node-metastasis (TNM) staging system is still under debate. The purpose of this study was to evaluate the prognostic efficacy of the 7th edition of the AJCC/UICC TNM staging system (focusing on $\mathrm{N}$ stage), in comparison with the 6th edition, at a single Eastern institution. Methods: We analyzed 1,435 patients with gastric cancer who underwent curative resection performed from September 1998 to August 2003 at the Memorial Jin-Pok Kim Korea Gastric Cancer Center. We analyzed the survival rate of the patients according to the AJCC/UICC 6th and 7th edi-
\end{abstract}




\section{Case Reports in Oncology}

Oh et al.: Prognostic Discrepancy of the 6th and 7th UICC N Classification for Lymph

Node Staging in Gastric Cancer Patients after Curative Resection

tions, and compared each stage, focusing on N stage. Results: Significant differences in the 5 -year survival rates were observed between the 6th and the 7th AJCC/UICC staging system. In the 6th edition staging system, the Kaplan-Meier curves discriminated each $\mathrm{N}$ stage significantly. In contrast, there was no difference in terms of survival curves for $\mathrm{N}$ stage according to the 7th edition, especially between N1 and N2: the Kaplan-Meier plots of survival curves between N1 (77.0\%) and N2 (78.1\%) stages overlapped significantly $(p<0.05)$. Conclusion: Although the 7th UICC staging system is a more detailed and sophisticated system in the T category, there was no prognostic significance between the pN1 and pN2 stages according to our data. Therefore, we suggest establishing a new UICC staging system taking into consideration the application of the $\mathrm{N}$ stage.

(C) 2017 The Author(s)

Published by S. Karger AG, Basel

\section{Introduction}

Although the survival rates of patients with gastric cancer have markedly improved owing to early diagnosis via nationwide screening programs as well as due to radical surgery in Eastern countries including Korea and Japan, gastric cancer remains the fourth leading cause of death in the world and the second-ranked cause of death among cancers in Korea [1,2].

Radical surgery for gastric cancer is the major key to curing patients with this disease [1, 3]. In gastric cancer, the depth of the primary tumor and the number of metastatic lymph nodes (LNs) are known to be the most important prognostic factors. The extent of LN metastasis, in particular, has been considered a key independent prognostic factor. Based on the tumor-node-metastasis (TNM) system, treatment plans are determined and the outcomes are predicted for gastric cancer. There have been several changes to the American Joint Committee on Cancer (AJCC) TNM staging system. In the 4th edition of the AJCC TNM staging system, $\mathrm{N}$ stage was described based on the extent of anatomical lymphatic spread (less than or greater than $3 \mathrm{~cm}$ from the primary tumor). However, according to the 5th and 6th editions of the AJCC TNM system, $\mathrm{N}$ stage was classified based on the number of metastatic LNs [4]. In 2010, the AJCC/Union Internationale contre le Cancer (AJCC/UICC) revised the staging systems of the TNM classification for gastric cancer [5]. Major changes were adopted in the 7th edition, including revisions of the $\mathrm{pT}$ and $\mathrm{pN}$ classifications. The subgroups pT2a and pT2b were reclassified in the new edition as pT2 and pT3, respectively. Moreover, major modifications were adopted with respect to the number of metastatic LNs considered for prognosis. The earlier classification of pN1 stage (1-6 involved regional LNs) was revised to pN1 (1-2 involved regional LNs) and pN2 (3-6 involved regional LNs), and the earlier pN3 subgroups $(>15)$ were merged into a new pN3 subgroup ( $>6$ involved regional LNs). As a whole, the number of LNs for staging $\mathrm{N}$ category was significantly decreased compared with that described in the 6th edition of the TNM staging system (where at least 16 resected LNs were required). However, in the new staging system the cutoff number of LNs is not included [6]. Since the revision of the staging system of gastric cancer, a large amount of literature concerning the prognostic implications of the 7th edition has been published. Most of the studies found the 5-year survival rates for each edition of the AJCC/UICC TNM staging systems to be significantly different from each other from the viewpoints of Eastern and Western countries. Some reports described that the 7 th staging system was better than the 6 th $[7$, 


\section{Case Reports in Oncology}

8]. On the other hand, some published reports have arrived at very different conclusions [2]. Therefore, we evaluated the 7th AJCC/UICC TNM staging system based on collected data from a single center regarding 1,435 gastric cancer patients who were surgically treated by a single surgeon in order to compare the prognostic implications of each edition of the TNM system, focusing on $\mathrm{N}$ stage.

\section{Patients and Methods}

We analyzed our prospectively collected database of 1,435 consecutive patients who were treated at the Memorial Jin-Pok Kim Korea Gastric Cancer Center, Seoul Paik Hospital, Inje University, from September 1998 to August 2003. All patients underwent radical subtotal or total gastrectomy according to the guidelines of the Japanese Research Society for the Study of Gastric Cancer, including radical D2 LN dissection, performed by the deceased professor Jin-Pok Kim. Survival time was calculated from the day of surgery to the last day of follow-up or the date of tumor-related death. Overall survival was defined as the time between the date of surgery and the date of death. The last day of follow-up was January 10, 2009 , and the average length of follow-up was 82 months (55-123 months). Statistical analysis was performed using SPSS statistics software (version 19.0; SPSS Inc., Chicago, IL, USA). The Kaplan-Meier method was used to estimate time-dependent survival probabilities. The log-rank test was used for statistical comparisons of survival curves. Cox proportional hazards models were used to investigate the multivariate association of clinical parameters and survival. All statistical data were considered significant when the $p$ value was less than 0.05 .

\section{Results}

\section{Patient Characteristics}

We included 1,435 patients who underwent curative resection in this retrospective analysis. This study group included $983(68.5 \%)$ male patients and 452 (31.5\%) female patients, resulting in a male-to-female ratio of 2.29:1. The mean age of the patients was 56.7 years, with a range of 21-85 years. The total number of resected LNs was 69,395 , and the mean number of resected LNs was 48 (5-130). According to the 6th edition staging system, the number of metastatic LNs was 0 (N0) in 662 patients (46.1\%), 1-6 (N1) in 326 patients (22.7\%), 7-15 (N2) in 195 patients (13.6\%), and more than $15(\mathrm{~N} 3)$ in 252 patients $(17.5 \%)$. According to the 7th edition, the number of metastatic LNs was as follows: 0 (N0) in 662 patients (46.1\%), 1-2 (N1) in 147 patients $(10.2 \%), 3-6(\mathrm{~N} 2)$ in 183 patients $(12.7 \%), 7-15$ (N3a) in 191 patients $(13.3 \%)$, and more than $15(\mathrm{~N} 3 \mathrm{~b})$ in 252 patients $(17.5 \%$; Table 1$)$. The overall 5-year survival rate was $69.6 \%$. The comparative study of 5 -year survival rates according to the 6 th and 7 th editions of the $\mathrm{N}$-stage system showed the following results (Table 1).

According to the 6th edition, the 5-year survival rates were as follows $(p<0.001)$ : stage Ia (92.7\%), Ib (89.1\%), II (82.4\%), IIIa (67.5\%), IIIb (41.5\%), and IV (24.2\%). Based on the 7 th edition, the 5-year survival rates for each stage were the following: stage Ia (95.6\%), Ib (84.2\%), IIb (75.7\%), IIIa (66.0\%), IIIb (35.4\%), and IIIc (35.2\%), respectively. In the N stage 


\section{Case Reports in Oncology}

of the 6th edition, the 5-year survival rates of patients were as follows ( $p<0.001$; Fig. 1): N0 (92.8\%), N1 (77.2), N2 (50.3\%), and N3 (24.9\%). In the 6th edition staging system, the Kaplan-Meier curves discriminated each $\mathrm{N}$ stage significantly. In contrast, survival curves for $\mathrm{N}$ stage according to the 7th edition were as follows $(p<0.001)$ : N0 (92.8\%), N1 $(77.0 \%), \mathrm{N} 2$ (78.1\%), N3a (54.1\%), and N3b (30.6\%). There was no difference in terms of survival between N1 and N2: the Kaplan-Meier plots of survival curves between N1 and N2 stages overlapped significantly $(p<0.05)$ (Fig. 2$)$.

\section{Prognostic Value}

The statistical assessment of the predictive value of the two scoring systems revealed no superiority of the 7th edition of the staging system (UICC 2010) when compared with the 6th edition (2002) concerning LN stage. The $\chi^{2}$ score for the 6th edition was higher than that in the 7 th edition, indicating that the $\mathrm{N}$ classification in the 6th edition has better discriminatory ability in terms of survival than that of the 7th edition staging system (Fig. 2).

\section{Discussion}

In this study, we sought to evaluate the usefulness of the 7th AJCC/UICC TNM staging system in patients with gastric cancer. According to our analysis, in the 6th edition of the AJCC TNM staging system, the Kaplan-Meier curves discriminated each N stage significantly. However, according to the 7th edition, the Kaplan-Meier plots of survival curves between N1 and N2 stages overlapped significantly $(p<0.05)$. There are many reports describing limitations of the 7th edition of the AJCC/UICC TNM staging system. In a Western study group, Patel et al. [8] reported that in a group of 1,905 patients from the American population, the 7 th edition of the staging system distinguished outcomes poorly, and the authors proposed a revised grouping allocation. A report including 1,767 European gastric cancer patients claimed that the 7 th edition had become more complex, without predictability for overall survival in the Western population, and supported some revision of the 7th edition of the AJCC/UICC TNM staging system [9]. Marano et al. [2] showed the prognostic superiority of the 6th edition $\mathrm{N}$-stage classification system in terms of homogeneity, discriminative ability, and monotonicity of gradients compared with the N classification of the 7th TNM staging, which showed similar survival curves between N2 and N3a, and the authors provided references for the revision of a future edition of the AJCC/UICC system for gastric cancer staging. Likewise, another Italian group reported that the 7th UICC N stage does not seem to be superior to the 6th UICC N stage in patients with fewer than 6 positive LNs [10]. In an Asian study group, Jung et al. [11] proposed a new pN classification due to the low power discrimination between pN1 and pN2, despite the fact that the 7th edition of the TNM classification proved to show significant differences in prognosis for each stage. Another study of 1,799 patients from South Korea did not show significant differences in the survival rates between each stage, especially for stages IB, IIA, and IIB, because of inappropriate $\mathrm{N}$ classification, and the authors suggested a new TNM system with a different $\mathrm{N}$ classification [12].

However, some other reports support the usefulness of the 7th edition of the AJCC/UICC TNM staging system for gastric cancer. An Eastern study with 1,503 gastric cancer patients reported that the Kaplan-Meier plot for the 6th edition did not show discriminative survival 


\section{Case Reports in Oncology}

Oh et al.: Prognostic Discrepancy of the 6th and 7th UICC N Classification for Lymph

Node Staging in Gastric Cancer Patients after Curative Resection

curves for N2 versus N3, but the 7th edition Kaplan-Meier plot showed discriminative survival curves between each $\mathrm{N}$ stage; therefore, the authors concluded that the 7th edition of the AJCC/UICC TNM staging system is superior to the 6th edition [13].

Another Eastern study in China with 456 gastric cancer patients also reported that there was a significant difference in the 5-year survival rates between the $\mathrm{N} 1$ and $\mathrm{N} 2$ substages of the 7th AJCC/UICC N stage. However, the authors found no significant prognostic difference between the N2 and N3 substages of the 5th/6th AJCC/UICC N stage, and concluded that the 7th AJCC/UICC $\mathrm{N}$ stage was preferable to the 5 th/6th $\mathrm{N}$ stage and the Japanese $\mathrm{N}$ stage for prognostic prediction [14]. The studies based in China can be discussed here because the sample sizes of the N3a and N3b groups are much smaller than those in other studies. Moreover, the authors did not perform subgroup analysis between N3a and N3b, nor did they comment on the mean number of total retrieved LNs [14]. Unwarranted stage migration may appear as a result of the lower power of discrimination in the 7 th $\mathrm{N}$ stage, especially between N1 and N2, in cases of fewer numbers of total retrieved LNs. Warneke et al. [15] reported that the 7 th edition of the staging system is superior to the 6th edition. However, more than $50 \%$ of the patients analyzed by them had fewer than 15 affected LNs. Moreover, this study did not show significant differences in the survival rates between N1 and N2 in patients with fewer than 6 metastatic LNs, although, on the other hand, there was distinct discrimination of survival curves between N3a (7-15) and N3b $(>15)$ based on the 15 retrieved LNs. The overlap between the N1 and N2 stages in the Kaplan-Meier plots of survival curves for the 7th edition might have been influenced by the small cutoff number of LNs between the $\mathrm{N} 1$ and $\mathrm{N} 2$ stages out of the average 46 resected LNs in our institute.

Although there have been controversies related to the extent of LN dissection to cure stomach cancer, Kim et al. [1] reported that radical LN dissection with more than 26 resected LNs improved survival in patients with stage II and IIIa disease per the 6th edition staging system (with emphasis on extensive lymphadenectomy) out of an average number of 31 dissected LNs. The paradoxical results concerning the comparison of the 6th and 7th edition staging systems between each of the reports might be due to the number of LNs analyzed. In a randomized nationwide Dutch trial, D2 lymphadenectomy was associated with lower locoregional recurrence and gastric cancer-related death rates than D1 resection. Therefore, the authors emphasized that D2 dissection is the standard surgical approach for resectable gastric cancer [16]. Using data from a large US population database, Smith et al. [17] reported that there is an optimal number of LNs to be examined for proper staging and survival prediction. They analyzed the Surveillance, Epidemiology, and End Results (SEER) data and concluded that the greater the number of LNs examined, the better the resulting postgastrectomy survival of patients with T1-3N0-1 gastric cancer. They also found that the trend towards superior survival outcome could be followed up to LN counts greater than 40. Survival prediction was more accurate when at least 15 nodes were examined, which is essential for an adequate $\mathrm{N}$-stage classification. However, this condition for the 7 th $\mathrm{N}$-stage classification staging system leads to stage migration and misunderstanding of the disease when few LNs have been examined $[17,18]$. Therefore, some studies have been carried out on the ratio of metastatic LNs, which could be a useful prognostic indicator in combination with the TNM staging system $[1,19,20]$. A small LN ratio despite the large number of metastatic LNs might be due to the extended LN dissection. If the LN dissection were less extensive, the LN ratio would have been higher with poor prognosis. The evaluation of the LN ratio might be influ- 


\section{Case Reports in Oncology}

Case Rep Oncol 2017;10:57-65

DOI: $10.1159 / 000455189$

(

(C) 2017 The Author(s). Published by S. Karger AG, Basel www.karger.com/cro

Oh et al.: Prognostic Discrepancy of the 6th and 7th UICC N Classification for Lymph

Node Staging in Gastric Cancer Patients after Curative Resection

enced by the extent of dissection performed by the surgeon. For accurate pathologic evaluation, the number of metastatic LNs out of dissected LNs should be included to calculate the ratio, and the LN ratio can then be used as a prognostic factor for stomach cancer: a sufficient amount of LNs should be retrieved [19].

Some limitations of the present study should be considered. First, this study included a relatively small sample size generated from a single institution experience, compared with a worldwide gastric cancer collaboration database. However, this study showed significant differences in survival curves for both TNM staging systems except the 7 th $\mathrm{N}$ classification. Second, the pT stage could not be reevaluated by pathologists because this was a retrospective study, and thus an unwarranted stage migration phenomenon could occur.

\section{Conclusions}

Despite the limitations of our analysis, the 6th UICC N-stage classification system was superior to the 7th classification staging system. We suggest further revisions to develop a new TNM staging system with appropriate $\mathrm{N}$-stage classification.

\section{Statement of Ethics}

Approval for this study was obtained from the Institutional Review Board of the Ethics Committee of the Haeundae Paik Hospital, Inje University College of Medicine, Korea. All procedures followed were in accordance with the ethical standards of the responsible committee on human experimentation (institutional and national) and with the Helsinki Declaration of 1975. Informed consent was obtained from all patients before being included in the study.

\section{Disclosure Statement}

None of the authors has any financial conflicts to disclose associated with this study.

\section{References}

1 Kim J, Lee J, Kim S, Yu H, Yang H: Clinicopathologic characteristics and prognostic factors in 10,783 patients with gastric cancer. Gastric Cancer 1998;1:125-133.

2 Marano L, Boccardi V, Braccio B, et al: Comparison of the 6th and 7th editions of the AJCC/UICC TNM staging system for gastric cancer focusing on the " $\mathrm{N}$ " parameter-related survival: The monoinstitutional NodUs Italian study. World J Surg Oncol 2015;13:1.

3 Kim JP, Kwon OJ, Oh ST, Yang HK: Results of surgery on 6,589 gastric cancer patients and immunochemosurgery as the best treatment of advanced gastric cancer. Ann Surg 1992;216:269-278; discussion 278-279.

4 Greene FL, Balch CM, Fleming ID, et al: AJCC Cancer Staging Handbook: TNM Classification of Malignant Tumors. Chicago, Springer Science and Business Media, 2002.

5 Washington K: Of the AJCC cancer staging manual: stomach. Ann Surg Oncol 2010;17:3077-3079. 


\section{Case Reports in Oncology}

Oh et al.: Prognostic Discrepancy of the 6th and 7th UICC N Classification for Lymph

Node Staging in Gastric Cancer Patients after Curative Resection

6 Sobin LH, Gospodarowicz MK, Wittekind C: TNM Classification of Malignant Tumours. Oxford, Wiley, 2011.

7 Zhang J, Zhou Y, Jiang K, et al: Evaluation of the seventh AJCC TNM staging system for gastric cancer: a meta-analysis of cohort studies. Tumor Biol 2014;35:8525-8532.

$>8$ Patel MI, Rhoads KF, Ma Y, et al: Seventh edition (2010) Of the AJCC/UICC staging system for gastric adenocarcinoma: is there room for improvement? Ann Surg Oncol 2013;20:1631-1638.

9 Reim D, Loos M, Vogl F, et al: Prognostic implications of the seventh edition of the international union against cancer classification for patients with gastric cancer: the Western experience of patients treated in a single-center European institution. J Clin Oncol 2013;31:263-271.

10 Rausei S, Dionigi G, Ruspi L, et al: Lymph node staging in gastric cancer: new criteria, old problems. Int J Surg 2013;11(suppl 1):S90-S94.

11 Jung H, Lee HH, Song KY, et al: Validation of the seventh edition of the American Joint Committee on Cancer TNM staging system for gastric cancer. Cancer 2011;117:2371-2378.

12 Yoon HM, Ryu KW, Nam BH, et al: Is the new seventh AJCC/UICC staging system appropriate for patients with gastric cancer? J Am Coll Surg 2012;214:88-96.

13 Wang W, Sun X, Li C, et al: Comparison of the 6th and 7th editions of the UICC TNM staging system for gastric cancer: results of a Chinese single-institution study of 1,503 patients. Ann Surg Oncol 2011;18:1060-1067.

14 Deng J, Liang H, Sun D, et al: Suitability of 7th UICC N stage for predicting the overall survival of gastric cancer patients after curative resection in china. Ann Surg Oncol 2010;17:1259-1266.

15 Warneke VS, Behrens HM, Hartmann JT, et al: Cohort study based on the seventh edition of the TNM classification for gastric cancer: proposal of a new staging system. J Clin Oncol 2011;29:2364-2371.

16 Songun I, Putter H, Kranenbarg EM, et al: Surgical treatment of gastric cancer: 15-year follow-up results of the randomised nationwide Dutch D1D2 trial. Lancet Oncol 2010;11:439-449.

-17 Smith DD, Schwarz RR, Schwarz RE: Impact of total lymph node count on staging and survival after gastrectomy for gastric cancer: data from a large US population database. J Clin Oncol 2005;23:71147124.

18 Karpeh MS, Leon L, Klimstra D, Brennan MF: Lymph node staging in gastric cancer: is location more important than number? An analysis of 1,038 patients. Ann Surg 2000;232:362-371.

$\checkmark 19$ Ema A, Yamashita K, Sakuramoto S, et al: Lymph node ratio is a critical prognostic predictor in gastric cancer treated with S-1 chemotherapy. Gastric Cancer 2014;17:67-75.

-20 Ahn JR, Jung M, Kim C, et al: Prognosis of pN3 stage gastric cancer. Cancer ResTreat 2009;41:73-79. 

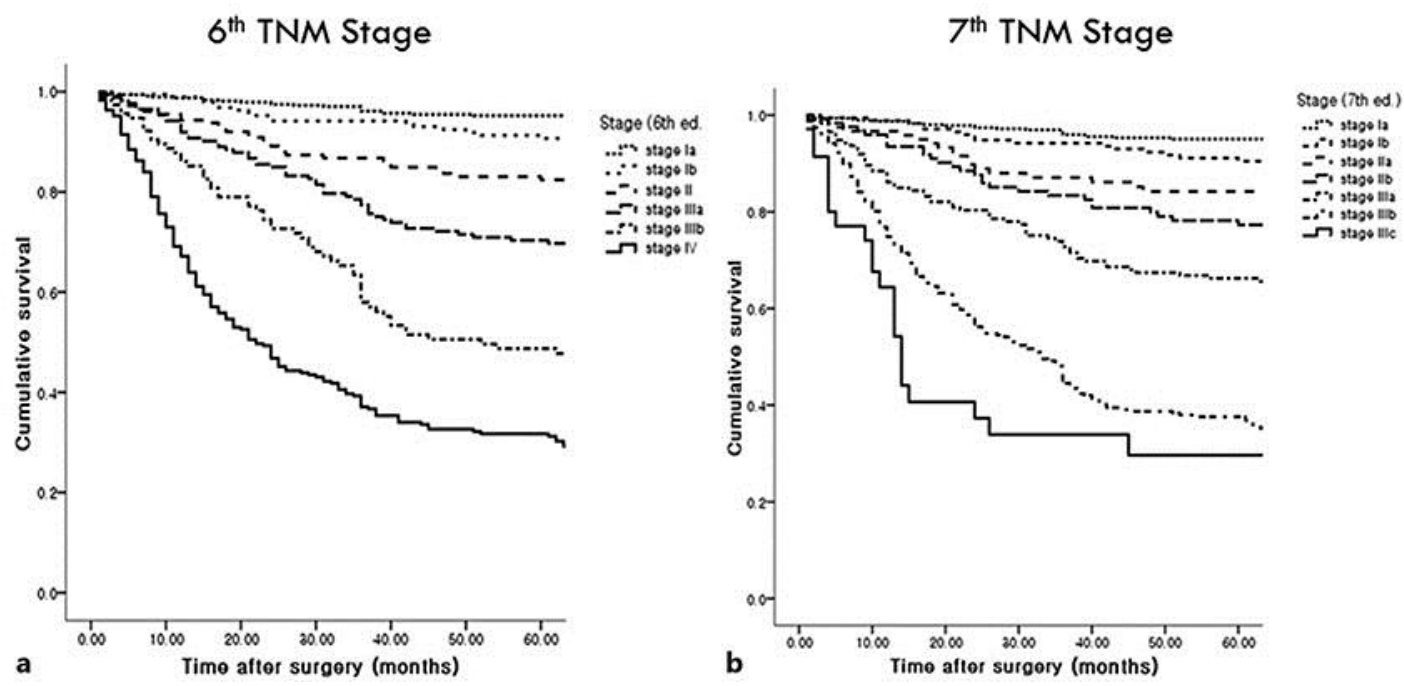

Fig. 1. Comparison of 5-year survival rates according to each stage of the 6 th and 7 th editions of the AJCC/UICC TNM staging system. a According to the 6th edition, 5-year survival rates are as follows ( $p<$ 0.001): stage Ia (92.7\%), Ib (89.1\%), II (82.4\%), IIIa (67.5\%), IIIb (41.5\%), and IV (24.2\%). b According to the 7 th edition, 5-year survival rates for each stage are as follows: stage Ia (95.6\%), Ib (84.2\%), IIb (75.7\%), IIIa (66.0\%), IIIb (35.4\%), and IIIc (35.2\%).
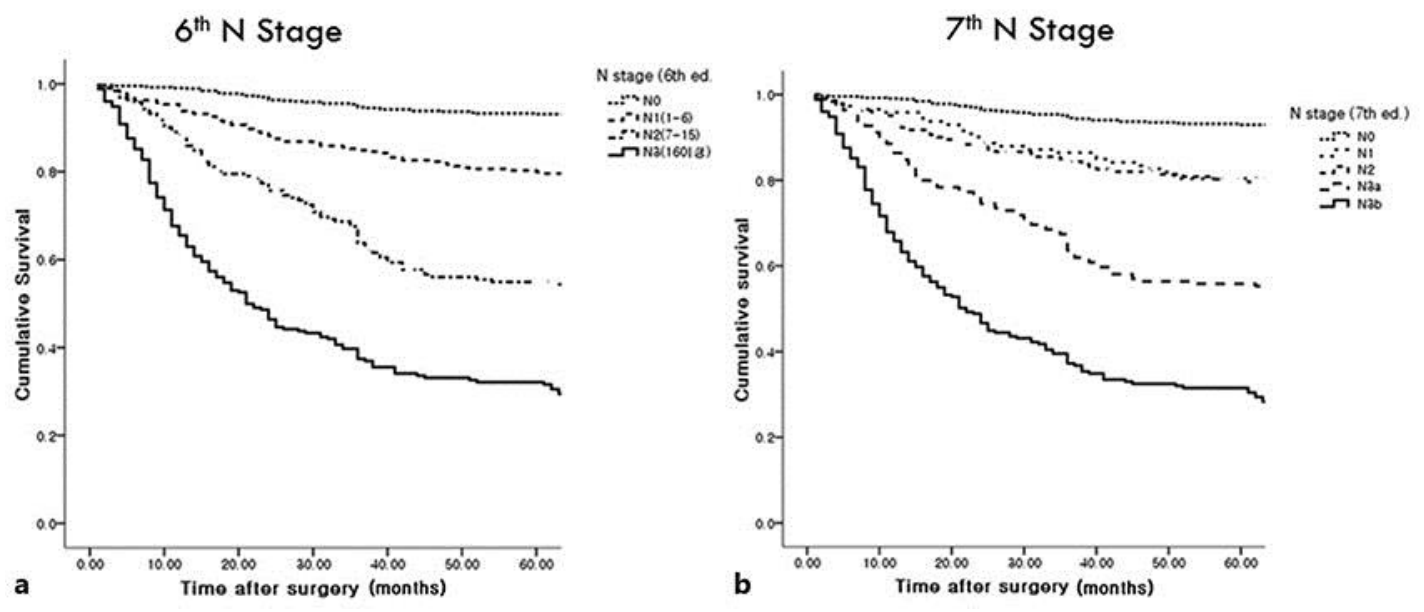

Fig. 2. Comparison of 5-year survival rates according to each stage of the 6th (a) and 7th (b) editions of the AJCC/UICC TNM staging system. A significant overlap is noted within the individual components of pN1 and $\mathrm{pN} 2$ in the 7 th edition of the staging system. The $\chi^{2}$ score in the 6th edition was higher than that in the 7 th edition, indicating that the $\mathrm{N}$ classification in the 6th staging system has better discriminatory ability in terms of survival than that of the 7 th staging system. 
Oh et al.: Prognostic Discrepancy of the 6th and 7th UICC N Classification for Lymph

Node Staging in Gastric Cancer Patients after Curative Resection

Table 1. Univariate survival analysis of clinicopathologic variables in 1,435 patients with gastric cancer

\begin{tabular}{|c|c|c|c|}
\hline $\begin{array}{l}\text { Clinicopathologic } \\
\text { features }\end{array}$ & $\begin{array}{l}\text { Patients, } \\
\mathrm{n}(\%)\end{array}$ & $\begin{array}{l}\text { 5-year } \\
\text { survival } \\
\text { rate, \% }\end{array}$ & $p$ value \\
\hline Age & & & $<0.001$ \\
\hline$<40$ years & $155(10.8)$ & 80.2 & \\
\hline $41-60$ years & $586(40.8)$ & 74.3 & \\
\hline$>60$ years & $694(48.4)$ & 61.5 & \\
\hline Gender & & & 0.834 \\
\hline Male & $983(68.5)$ & 70.6 & \\
\hline Female & $452(31.5)$ & 71.5 & \\
\hline Location of tumor & & & 0.020 \\
\hline Upper $1 / 3$ & $197(13.7)$ & 50.9 & \\
\hline Middle $1 / 3$ & $385(26.8)$ & 80.5 & \\
\hline Lower $1 / 3$ & $853(59.3)$ & 71.8 & \\
\hline Tumor size & & & $<0.001$ \\
\hline$<5 \mathrm{~cm}$ & $646(44.9)$ & 86.8 & \\
\hline$\geq 5 \mathrm{~cm}$ & $789(55.0)$ & 51.9 & \\
\hline Histology & & & $<0.001$ \\
\hline Differentiated & $619(43.1)$ & 73.8 & \\
\hline Undifferentiated & $816(56.9)$ & 63.1 & \\
\hline Lauren classification & & & $<0.001$ \\
\hline Intestinal & $704(49.1)$ & 75.2 & \\
\hline Diffuse & $731(50.1)$ & 66.5 & \\
\hline TNM classification by 6th & & & $<0.001$ \\
\hline \multicolumn{4}{|l|}{ AJCC/UICC system (N stage) } \\
\hline N0 (0) & $662(46.1)$ & 92.8 & \\
\hline $\mathrm{N} 1(1-6)$ & $326(22.7)$ & 77.2 & \\
\hline $\mathrm{N} 2(7-15)$ & $195(13.6)$ & 50.3 & \\
\hline N3 $(>15)$ & $252(17.5)$ & 24.9 & \\
\hline TNM classification by 7th & & & $<0.001$ \\
\hline \multicolumn{4}{|l|}{ AJCC/UICC system (N stage) } \\
\hline No $(0)$ & $662(46.1)$ & 92.8 & \\
\hline $\mathrm{N} 1(1-2)$ & $147(10.2)$ & 77.0 & \\
\hline N2 $(3-6)$ & $183(12.7)$ & 78.1 & \\
\hline N3a $(7-15)$ & $191(13.3)$ & 54.1 & \\
\hline $\mathrm{N} 3 \mathrm{~b}(>15)$ & $252(17.5)$ & 30.6 & \\
\hline Type of surgery & & & $<0.001$ \\
\hline Radical subtotal gastrectomy & $976(68.0)$ & 76.2 & \\
\hline Radical total gastrectomy & $446(31.1)$ & 58.4 & \\
\hline Proximal subtotal gastrectomy & $13(0.9)$ & 69.2 & \\
\hline
\end{tabular}

